\title{
Théologiques
}

\section{Option pour les pauvres : bilan et enjeux}

\section{Gustavo Gutiérrez}

Volume 1, numéro 2, octobre 1993

Les textes fondateurs. Entre autorité et liberté

URI : https://id.erudit.org/iderudit/602394ar

DOI : https://doi.org/10.7202/602394ar

Aller au sommaire du numéro

Éditeur(s)

Faculté de théologie de l'Université de Montréal

ISSN

1188-7109 (imprimé)

1492-1413 (numérique)

Découvrir la revue

Citer cet article

Gutiérrez, G. (1993). Option pour les pauvres : bilan et enjeux. Théologiques, 1(2), 121-134. https://doi.org/10.7202/602394ar d'utilisation que vous pouvez consulter en ligne.

https://apropos.erudit.org/fr/usagers/politique-dutilisation/ 


\title{
Option pour les pauvres: bilan et enjeux. 1
}

\author{
Gustavo GUTIÉRREZ \\ Centre Bartolome de Las Casas \\ Pérou
}

Je voudrais tout d'abord remercier la Faculté de théologie de l'Université de Montréal de l'honneur qu'elle me fait en me remettant ce doctorat honorifique. Comment vous remercier pour ce geste gratuit que, par définition, on ne mérite pas!

Je suis particulièrement heureux d'être ici de nouveau, à Montréal. En effet, il y a vingt-six ans, à l'été de 1967, pendant l'Exposition universelle et pendant la visite de De Gaulle, j'ai donné un cours de deux semaines à votre Faculté. Le thème portait sur l'Église et les problèmes de la pauvreté, le même thème que vous m'offrez de traiter ce soir. J'en avais profité alors -c'était à peine deux ans après le Concile - pour travailler surtout sur la signification biblique et théologique de la pauvreté. C'était une année avant que n'apparaisse l'expression théologie de la libération. Je puis vous dire que le contenu de cette théologie a pris naissance dans ce cours donné a Montréal. C'est pourquoi il m'est très agréable de me retrouver avec vous.

1 Texte de la conférence prononcée le 16 septembre 1993, dans le cadre des fêtes du 25 e anniversaire de l'implantation de la Faculté de théologie sur le campus de l'Université de Montréal. Gustavo Gutiérrez a donné son accord à la publication de ce texte. Des problèmes de communication ont empêché qu'il en vérifie la dernière version. Nous nous en excusons auprès de l'auteur. 


\section{Un souvenir}

Permettez-moi de vous faire part d'un souvenir. J'ai eu l'occasion d'aller à la quatrième session du Concile Vatican II. Les deux derniers jours avant la clôture, j'avais travaillé avec un évêque chilien, qui était alors Président du Conseil épiscopal pour l'Amérique latine (CELAM), Monseigneur Manuel Larrain. Par son intermédiaire, j'avais reçu une invitation à assister à la clôture du Concile. Mais je n'ai pas eu la force d'y aller. Pendant ces deux jours, les 7 et le 8 décembre 1965, j'ai fait une retraite seul, chez moi, et c'est là que j'ai eu l'occasion d'écouter à la radio l'un des plus beaux discours de Paul VI sur la signification du Concile. Je vivais alors, et j'emprunte une expression française, des 'sentiments mêlés'. D'une part, j'étais très heureux du Concile où s'était exprimée la théologie que j'avais étudiée et à laquelle je m'identifiais. D'autre part, je ressentais un très grand malaise: les grands défis de mon peuple latino-américain n'étaient pas présents. Le regard sur la pauvreté n'a pas été très présent au Concile, ni la situation très conflictuelle que l'on vivait en Amérique latine. Mon côté européen, si l'on veut, était content; mais mon côté latino-américain, plus métis ou indien, était plutôt confus, en attente d'autre chose. Et je me souviens très bien que c'est à partir de ces deux jours d'expérience spirituelle, s'il faut fixer une date, que j'ai commencé à prendre une certaine distance avec la théologie que j'avais travaillée et lue avec ferveur. Je venais de vivre mes premières années de travail pastoral et j'avais senti en Amérique latine et particulièrement dans mon pays, le Pérou, une réalité qui ne correspondait pas exactement aux résultats du Concile. Au-delà des expériences personnelles, c'est de cela dont je voudrais vous parler tel que j'y réfléchis aujourd'hui.

L'intervention comportera trois parties. La première consistera en un rapide parcours historique, de ce qu'on appelle aujourd'hui l'option préférentielle pour les pauvres. Dans ma deuxième partie, je voudrais saisir avec vous le sens de cette expression. Enfin, une troisième partie, toute courte, portera sur ce qu'on a commencé dernièrement à appeler - et vous verrez que c'est vieux - la nouvelle évangélisation.

\section{Parcours historique}

Les discours de Jean XXIII autour du Concile peuvent se résumer en une question fondamentale et trois grandes intuitions. La question était: comment être capable de dire aujourd'hui "que ton règne arrive?" 
C'était l'inquiétude profonde du pape à partir de laquelle il a parlé de trois préoccupations majeures, en particulier de la présence de l'Église aux trois mondes. En tout premier lieu, de l'ouverture au monde moderne. Je ne vais pas la développer longuement. Vous connaissez ces choses tellement bien. Les documents préparatoires du Concile n'allaient pas dans ce sens. Mais rappelez-vous, ils ont été rejetés, sauf celui sur la liturgie, parce qu'ils ne correspondaient pas aux intuitions de Jean XXIII. Au Concile, on disait, en blaguant, qu'ils avaient été rejetés grâce à "l'attaque rouge". Ce n'était pas du tout les communistes de l'époque, mais il s'agissait de sept cardinaux, dont le cardinal Liénart de Paris, qui avaient contribué au rejet des schémas préparatoires. Ces interventions ont permis la rédaction de la Constitution L'Église dans le monde de ce temps où le thème de l'ouverture au monde moderne est très présent.

La deuxième intuition fut celle de la présence de l'Église dans un autre monde: le monde chrétien, celui du dialogue oecuménique. Bien sûr, ce dialogue avait commencé depuis quelques décades avant le Concile, mais les Pères ont accepté cette intuition du pape et l'ont étendue au monde religieux en général. D'où la publication des documents sur les grandes religions de l'humanité. Le dialogue oecuménique avait été favorisé par la présence de quelques experts orthodoxes et protestants au Concile, dont Karl Barth.

Mais un mois avant le début du Concile, exactement le 11 septembre 1962, Jean XXIII a prononcé un discours dans lequel il a utilisé l'expression: “un autre point lumineux". Si vous avez une certaine familiarité avec les textes de Jean XXIII, vous aurez compris que chaque fois qu'il veut parler de quelque chose d'important, il parle de point lumineux, «ponto luminoso». Il parle d'un autre point lumineux, mais de grâce, ne cherchez pas avec des catégories cartésiennes quel était le premier. C'était une façon d'attirer l'attention sur ce qu'il veut dire. "L'Église est et veut être l'Église de tous, et particulièrement l'Église des pauvres". II a dit qu'en face de la pauvreté du monde, l'Église était (telle est la réalité) et l'Église voulait être (tel est le projet) l'Église de tous et, particulièrement l'Église des pauvres. On ne peut pas séparer "l'Église des pauvres" de "l'Église de tous".

\subsection{Le thème de la pauvreté au Concile}

Jean XXIII a proposé cette vision de l'Église au Concile comme un sujet qui s'imposait. Or les Pères du Concile qui avaient le plus 
d'influence venaient plutôt des pays du Nord, de l'Europe et de l'Amérique du Nord. Ils étaient pris par la question de l'ouverture au monde moderne. En fait, l'Église catholique n'avait pas encore accepté des dimensions comme la liberté, l'esprit démocratique, les sciences, les techniques, etc. La majorité des Pères étaient préoccupés aussi par le dialogue oecuménique, puisque dans les pays d'où ils revenaient, les églises, surtout protestantes, sont très fortes: Allemagne, États-Unis, Canada. Ils étaient tellement préoccupés par ces deux premiers points qu'ils ont un peu oublié le troisième: le souci de l'Église des pauvres dans le contexte que je viens de rappeler.

Dans les deux derniers jours de la première session du Concile, un ami très proche de Jean XXIII, le cardinal Lercaro, archevêque de Bologne et l'un des secrétaires du Concile, a fait une intervention dans laquelle il a affirmé: "le thème du Concile doit être l'évangélisation des pauvres, la pauvreté dans le monde». Et il a précisé - nous possédons le discours au complet - "ce n'est pas l'un des thèmes mais LE thème du Concile». Et de continuer: "Si l'on prend ce point-là, alors on pourra traiter de la question oecuménique et de l'ouverture au monde". Je vous prie de faire un petit effort. Imaginez que le Concile ait accepté la proposition de Lercaro, on aurait eu comme thème central, pour l'Église catholique, celui de la pauvreté dans le monde et de l'évangélisation des pauvres. Les Pères du Concile étaient émus en écoutant le cardinal Lercaro, mais comme vous le dites en français: "c'était trop beau pour être vrai”. Les préoccupations étaient autres. Deux autres cardinaux, très importants à l'époque, le cardinal Suenens, de Malines, en Belgique, et le cardinal Montini, celui qui, peu après, serait pape, ont alors plutôt souligné les deux premières intuitions, surtout la première, celle de l'ouverture au monde moderne.

On retrouve, dans les documents conciliaires, quelques passages sur la pauvreté. Ainsi, au numéro 8 de Lumen Gentium, on lit: "l'Église, comme son fondateur, vit dans la persécution et la pauvreté". Mieux encore, on lit dans l'un des meilleurs textes du Concile, le décret $A d$ Gentes sur la mission, au numéro 5: "L'Église doit prendre le chemin de la pauvreté pour annoncer l'Évangile". C'est bien, mais c'est peu. On peut comprendre, non pas l'absence, mais cette présence timide du thème de la pauvreté dans les documents conciliaires. Ne soyons pas anachronique. II serait facile aujourd'hui d'accuser le Concile. Mais les gens à l'époque connaissaient d'autres inquiétudes. Le sujet de la pauvreté, de l'Église des pauvres, n'avait pas encore, dans la conscience ecclésiale, l'importance qu'il a pris plus tard. 
Trois ans après le Concile, dans la cité colombienne de Medellin, a eu lieu une conférence épiscopale latino-américaine. C'était en 1968. A cette conférence, la troisième intuition du pape Jean XXIII était très présente. Cela n'est pas tellement étonnant, si vous constatez que les seuls pays qui soient à la fois majoritairement pauvres et chrétiens, se retrouvent en Amérique latine. En majorité, dis-je, car il y a des chrétiens en proportion importante en Afrique; mais pour des raisons historiques que nous connaissons, ils ne le sont pas dans des proportions si importantes. Ce n'est pas le cas de l'Asie où les chrétiens sont en minorité. Le seul continent, donc, où nous retrouvons pauvreté et foi chrétienne est l'Amérique latine. On y a repris cette troisième intuition de Jean XXIII. Je pense que la signification la plus importante de la conférence épiscopale de Medellin, a été le fait de voir une Église, celle d'Amérique latine, devenir adulte.

\subsection{Medellin et le sens de la pauvreté}

Les documents de Medellin comportent, selon un schéma qui vient de l'action catholique ouvrière, trois parties: vision de la réalité, réflexion et lignes pastorales. A Medellin, on a non seulement parlé de la paurreté latino-américaine, mais on a également signalé les causes de cette pauvreté. Voilà pourquoi on a parlé de libération: pauvreté et libération étaient les deux grands thèmes. On a voulu clarifier le sens du terme pauvreté. Une première précision concerne ce qu'on appelle la pauvreté réelle que, nous chrétiens, avons la mauvaise habitude d'appeler pauvreté matérielle. Cette vision est étrangère au vocabulaire biblique. Il est vrai que nous sommes tributaires de la philosophie grecque où règne la distinction entre matière et esprit. Mais la pauvreté matérielle, c'est la pauvreté réelle, concrète, celle de tous les jours. Cette pauvreté-là est un scandale, un mal, comme il est rappelé à Medellin.

Une deuxième précision a trait à ce qu'on a l'habitude d'appeler la pauvreté spirituelle, comprise non pas, en premier lieu, comme le détachement par rapport aux biens matériels, mais comme l'enfance spirituelle. Remettre nos vies dans les mains de Dieu: tel est le sens de la pauvreté spirituelle, sens le plus significatif dans la révélation chrétienne. Cette pauvreté permet de reconnaître Dieu comme amour, Père, Mère; de reconnaître les autres personnes comme des soeurs et des frères; accepter, donc, l'amour gratuit de Dieu. 
La pauvreté comme engagement, telle est la dernière précision apportée à Medellin: une solidarité avec les pauvres dans la lutte contre la pauvreté; un engagement pour les pauvres et contre la pauvreté.

Vous le savez, Medellin a été une voix très prophétique dans la vie de l'Église latino-américaine. Je ne prétends pas - et d'ailleurs ce n'est jamais ainsi - que l'ensemble de l'Église, toute l'Église latino-américaine a marché dans les pas de Medellin. Je le voudrais bien, mais vous n'êtes pas sans savoir les résistances à un engagement comme celui-là. Peu importe, c'était une voix significative qui a rendu possible bon nombre d'expériences et d'engagements. Cette vision a finalement été confirmée et ratifiée à Puebla où on a alors parlé de l'option préférentielle pour les pauvres. Cette expression comporte trois termes: pauvre, option et préférence. La "pauvreté" dont on parle ici est la pauvreté matérielle, la "préférence", la pauvreté spirituelle, et l'"option", l'engagement contre la pauvreté. A Santo Domingo, en 1992, entre bien d'autres points, on a ratifié cette vision. Santo Domingo a répété que l'Église latino-américaine avait une option ef différentes lignes pastorales, mais une option.

Je le souligne, parce que les documents préparatoires parlaient d'au moins, huit ou dix options préférentielles. Vous le savez, l'inflation amène toujours la dévaluation... Santo Domingo a réaffirmé une option et différentes lignes pastorales venant de cette option. J'arrête ici le parcours historique pour passer à l'explicitation du contenu de l'expression.

\section{L'option préférentielle pour les pauvres}

La meilleure façon de saisir les enjeux de l'expression est de la reprendre mot par mot: pauvreté, préférence et option.

\subsection{La pauvreté}

Quelle est cette pauvreté dont on parle lorsqu'on utilise l'expression: option préférentielle pour les pauvres? C'est très clair. La pauvreté comporte des dimensions économique, sociale, politique, mais elle est certainement plus que cela. En dernière analyse, en dernière instance, la pauvreté signifie mort, c'est la mort: mort injuste, mort prématurée des pauvres, mort physique. Vous le savez, 
ce n'est malheureusement pas seulement en Amérique latine, que les gens meurent des maladies que la médecine a déjà vaincues. II n'y a pas de raisons pour mourir du choléra; mais le choléra tue des personnes au Pérou, mon pays, et tue des gens dans d'autres endroits au monde, en Asie et ailleurs. Au Pérou, court une blague un peu sarcastique et un peu noire: le choléra est une maladie qui a fait l'option pour les pauvres. Le choléra tue les pauvres, et seulement les pauvres, parce que, si vous avez un peu d'argent, vous pouvez faire bouillir l'eau et le virus très fragile, meurt à soixante degrés celsius. Vous le constatez, il est facile de se protéger du choléra, mais les gens n'ont pas d'argent, même pas pour faire bouillir leur eau.

La pauvreté cause donc la mort physique, mais c'est aussi de mort culturelle dont il faut parler. Quand un peuple n'est pas apprécié, quand il est méprisé d'une manière ou d'une autre, d'une certaine façon, vous tuez les gens qui appartiennent à ce peuple. Nous avons en Amérique latine différentes races, différentes cultures et différentes langues. $\mathrm{Si}$ vous méprisez ces cultures et ces races, vous tuez les gens qui appartiennent à ces groupes sociaux. Les anthropologues aiment dire que la culture c'est la vie; si vous méprisez la culture, vous méprisez la vie. Une mort culturelle accompagne très souvent la mort physique. Ainsi, quand on ne reconnaît pas aux femmes la plénitude de leurs droits humains, elles ne sont plus des femmes au plan culturel. Elles ne le sont plus, parce qu'on ne les reconnaît pas pleinement comme des êtres humains avec les droits qui leur correspondent. La pauvreté signifie donc la mort. En disant cela, je n'ai pas voulu occulter la dimension sociale, économique ou politique; j'ai voulu plutôt insister sur le sens de cette pauvreté sociale, économique et politique pour vous dire que c'est finalement de la vie dont il est question. C'est pourquoi, dans les communautés chrétiennes, en Amérique latine, on parle fréquemment du Dieu de la Vie et on rejette son contraire: la mort physique, culturelle, comme d'autres manifestations d'égoïsme et de péché.

Qu'est-ce qu'on peut alors entendre par pauvre? Je pense qu'il n'y a pas une bonne définition. Je pense qu'une bonne manière de parler des pauvres, est de dire que les pauvres sont les in-signifiants, ceux qui ne comptent pas pour la société et très souvent pour les Églises chrétiennes. Le pauvre est celui qui peut attendre une semaine à la porte d'un hôpital pour voir un médecin. Un pauvre est celui qui n'a pas de poids social, que l'on vole par des lois injustes et qui n'a pas le pouvoir de parler pour changer cette situation. C'est l'In-signiflant qui n'a pas de poids économique, qui fait partie d'une race méprisée, 
culturellement marginalisée. Les pauvres sont socialement insignifiants, sauf devant Dieu. Ils sont toujours présents à travers des statistiques, mais ils n'ont pas de nom.

Je vous donne un exemple qui pourra vous sembler un peu cruel. J'ai participé aux funérailles de l'archevêque Roméro que je connaissais bien. On calcule que ce même jour, quarante personnes ont été tuées sur la place centrale de la cathédrale de San Salvador. Nous connaissons très bien le nom de Monseigneur Romero, parce qu'il était un archevêque, un grand homme bien sûr; mais nous ne connaissons pas les noms de ces quarante personnes qui sont mortes pour voir Romero une dernière fois. A cóté de moi, dans la cathédrale, je voyais cinq femmes mortes, une autre grièvement blessée, mais encore vivante; je l'ai entendue et j'ai pu faire quelque chose pour elle. Nous ne connaissons pas les noms de ces personnes, parce qu'elles sont pauvres tout autant dans leur vie que dans leur mort, et après leur mort. Vous comprenez qu'en disant cela, je ne dis rien contre Romero, mais je dis tout simplement que si vous occupez une place importante dans l'Église, qui est une institution, vous n'êtes pas exactement un In-signiflant. Mais nous ne connaissons pas les noms des pauvres. Ils demeurent des anonymes.

Un autre exemple. On me dit parfois: «vous, un latino-américain, vous êtes un pauvre". Je regrette. Je tâche d'être engagé avec les pauvres et j'habite dans un quartier pauvre, mais je ne suis pas un pauvre, parce que je ne suis pas un In-signifiant. Et je ne suis pas un in-signiflant parce que je suis prêtre. Il vaut mieux le dire clairement. Je tâche d'être avec les pauvres, mais comme prêtre et comme théologien dans mes temps libres, je mentirais en disant que je suis in-signiflant dans mon pays. Ce serait de la fausse modestie ou tout simplement de la modestie, parce que toutes les modesties sont fausses. Il vaut mieux reconnaître ce que l'on est et, avec une certaine humilité, essayer d'être proche des pauvres.

Enfin, pour terminer avec la pauvreté, il faut préciser ceci: si vous parlez des paurres, vous aurez probablement la réaction de personnes qui vous disent que vous êtes très généreux. Mais si vous parlez des raisons de la pauvreté, les gens peuvent se dire: "mais celui-là est-il chrétien, n'est-il pas plutôt politicien?" Au Congrès eucharistique de Philadelphie, un très grand personnage de l'Église d'aujourd'hui a dit en substance: “je viens d'un pays pauvre; vous êtes riches, vous les Américains, aidez-nous". Les participants ont applaudi pendant cinq minutes. Au mème Congrès, Dom Helder Camara a affirmé: “je viens 
d'un continent pauvre, l'Amérique latine. Notre pauvreté tient beaucoup aux corporations multinationales qui travaillent dans notre pays, et votre pays aide les corporations multinationales". On l'a applaudi d'une façon trés polie. II n'avait pas dit: "aidez-nous" mais "changez, tâchez de changer votre gouvernement".

\subsection{La préférence}

J'ai souvent rencontré des personnes qui trouvaient étrange l'emploi du terme "préférence". Ne serait-il pas préférable de dire tout simplement option pour les pauvres, préférence étant trop mou? Au contraire, il s'agit d'un terme chrétien qui contraint à préserver l'universalité de l'amour de Dieu dont personne n'est exclu. C'est seulement à l'intérieur de ce cadre de l'universalité que l'on peut comprendre la "préférence", c'est-à-dire "ce qui est premier". La Bible parle de la préiérence de Dieu pour les pauvres. Pourquoi, dans la Genèse, Dieu a-t-il préféré Abel à Caïn? II n'est pas dit qu'Abel était meilleur, une très bonne personne, et que Caïn était quelqu'un de mauvais. Mais Abel était le deuxième; il avait moins de poids humain. Dieu a préféré le sacrifice d'Abel au sacrifice de Caïn. Le péché de Caĩn est de n'avoir pas accepté la préférence de Dieu pour Abel. C'est pour cette raison qu'il l'a tué. Le refus de la préférence, c'est de ne pas comprendre qu'il faut tenir ensemble l'universalité de l'amour de Dieu et la préférence pour les plus pauvres. C'était déjà dans l'expression de Jean XXIII: “l'Église de tous et particulièrement l'Église des pauvres". Comme chrétiens nous ne pouvons pas dire: "il n'y a que les pauvres qui comptent". Cette attitude n'est pas chrétienne comme celle d'aimer tout le monde, alors qu'on n'aime personne. Tenir ensemble les deux aspects n'est pas facile. C'est un grand défi.

Mais pourquoi cette préférence? Vous pouvez facilement dire que c'est mon analyse sociale qui me permet de comprendre la pauvreté et préférer les plus pauvres. Cette analyse est certes utile, mais n'est pas suffisante. Ou encore, qu'il faut préférer les pauvres par compassion humaine. La compassion humaine est très importante, mais ce n'est pas la dernière raison. Ou encore dire: "si vous parlez tellement des pauvres et tellement fort, c'est parce que vous êtes latino-américain”. Je réponds toujours à ces personnes: "écoutez, tâchez de ne pas me comprendre trop vite: si je parle de la pauvreté c'est en premier lieu parce que je suis chrétien, en deuxième lieu, parce que je suis latinoaméricain. Si je parle de la pauvreté, c'est parce que je suis chrétien et si vous êtes chrétien, faites de même". La question géographique, si 
importante soit-elle, vient en second. Enfin, on entend dire que nous devons préférer les pauvres parce que les pauvres sont bons. II y en a, en effet, de très bons, mais aussi de très mauvais. Je ne vous conseille pas de venir me voir dans ma paroisse à deux heures du matin. C'est dangereux...

Nous devons préférer les pauvres, d'abord parce que Dieu est bon et qu'il préfère les plus oubliés, les opprimés, les pauvres, les abandonnés. Le fondement est en Dieu. La raison ultime, finale, de la "préférence", c'est le Dieu de notre foi. Cette affirmation est très engageante, parce que vous et moi croyons au même Dieu. Ce que je viens de dire vaut pour vous aussi; vous ne pouvez pas échapper à cette préférence pour les pauvres. Comment le faire? C'est une autre question. Mais si nous croyons au même Dieu, alors nous devons historiquement cheminer ensemble. La préférence vient de la bonté de Dieu et vient de l'amour gratuit de Dieu, la notion centrale du message évangélique. Dieu nous a aimé le premier. Notre vie, nos vies sont des réponses à cette initiative gratuite de Dieu. C'est la signification de la pauvreté spirituelle. On parle de gratuité; d'une gratuité comme celle qui caractérise l'amour des parents, d'une mère pour ses enfants.

Les grands mystiques, saint Jean de la Croix, par exemple, nous apprennent comment le Dieu gratuitement touché est au coeur de la vie spirituelle. Et cela ne s'oppose pas à l'engagement social et politique. Mais sans contemplation, sans prière, nous n'avons pas de vie chrétienne. Par ailleurs, sans solidarité avec les pauvres, nous n'avons pas de vie chrétienne non plus. Nous avons donc deux dimensions qu'il faut tâcher de tenir ensemble.

\subsection{Option}

On entend parfois ce genre d'observation: l'option pour les pauvres, c'est quelque chose que doivent faire les non pauvres. On oublie que les pauvres eux-mêmes doivent aussi faire l'option pour leurs soeurs et leurs frères. Parce qu'il arrive souvent que des pauvres ont fait une option non pour les pauvres, mais pour leur famille et pour eux-mêmes. L'option est plutôt une exigence libre. C'est une décision qui concerne tous les chrétiens, car tous doivent faire cette option. 


\section{La nouvelle évangélisation}

Si un jour quelqu'un me demandait: quelle est la perspective la plus importante dans la théologie que vous faites en Amérique latine, celle qu'on appelle la théologie de la libération? Je répondrais que c'est l'option préférentielle pour les pauvres. La théologie de la libération peut disparaître, pourvu que cette option demeure. Jusqu'à l'âge de quarante ans, je n'ai jamais parlé de la théologie de la libération. J'étais alors chrétien, ou je tâchais de l'être; et j'espère l'être tout autant après la théologie de la libération. Je ne crois pas à la théologie de la libération, je crois en Jésus Christ. Une théologie c'est un moyen pour comprendre ma foi. Ce n'est pas un article de ma foi, de mon credo. La théologie a une place importante mais à sa place.

Je me souviens très bien d'un jésuite colombien qui s'est approché de moi un jour, en me disant qu'il devait faire une thèse de théologie à Rome. Je voudrais, me disait-il, "faire ma thèse sur la théologie de la libération". La théologie de la libération est très utile aussi pour faire des thèses de doctorat. C'est pourquoi, quand on me demande, qu'est-ce que la théologie de la libération a apporté à l'Église? Je réponds: "beaucoup de docteurs". Ce Jésuite a pris un papier et un crayon, en me disant: "Peux-tu me dire les grands thèmes de la théologie de la libération?" Je lui ai répondu: "les thèmes sont: Dieu, la Trinité, la liberté, les sacrements, l'Église, la grâce, les péchés, la Vierge Marie». II m'a dit: "est-ce que tu te moques de moi?" Je lui ai répondu: “non, mais toi tu te moques de moi, n'est-ce pas?" Le point de départ de toute théologie c'est la révélation; les sujets sont les mêmes. Les théologies sont différentes dans le rapport aux mêmes thèmes, pas par le thème. Dans la façon de traiter un thème, il y a eu des différences en théologie au cours des deux mille ans. Je ne pense pas que la théologie de la liberation soit quelque chose de permanent. Aucune théologie ne doit l'être, et c'est même mauvais une théologie qui dure trop longtemps. On en a eu l'expérience dans l'Église. S'il reste cette préférence pour les pauvres, nous aurons gagné quelque chose d'important, parce qu'elle est profondément liée à la révélation dans la Bible. Ce sera le mérite de la théologie de l'Église latino-américaine d'avoir réappris au monde cette option.

L'option préférentielle pour les pauvres, c'est l'axe de ce qu'on appelle la nouvelle évangélisation. Vous connaissez l'expression; elle est à la mode. Ce que l'on sait peut-être moins c'est qu'elle se trouvait dans les documents de Medellin en 1968. Le document parle du besoin, en 
Amérique latine, d'une nouvelle évangélisation. Ce fut une sorte de prise de conscience celle d'être en train de vivre un moment historique différent, dans un contexte social et culturel autre qui demande de nouveaux schèmes pour annoncer l'Évangile. Une nouvelle évangélisation exige une nouvelle vision du monde. Autrement, cette expression risque de se vider de sa nouveauté. Dans mon continent, on parle depuis vingtcinq ans de cette nouvelle évangélisation.

Je ne sais s'il se trouve des latino-américains parmi vous et des personnes qui ont travaillé et qui travaillent là-bas. Quant à moi, je pense que l'Église latino-américaine vit son moment historique le plus riche. Riche, ne veut pas dire facile, car nous avons une richesse douloureuse: des martyrs et plusieurs chrétiens ont donné leur vie pour l'option préférentielle en faveur des pauvres. C'est le cas de Romero, évêque à Salvador, d'Angeli en Argentine, et d'Ellacuria à Salvador. Quand une Église est capable d'avoir parmi ses membres des gens qui donnent leur vie de cette façon, il y a quelque chose d'important qui arrive à cette Église. Il y a des personnes assassinées, et tant d'autres gens qui travaillent d'une façon anonyme et que personne ne connaît parfois à l'extérieur.

Les théologiens de la libération appartiennent, eux, à un monde connu, comme représentant de l'Église latino-américaine.

Parce que, comme théologiens, nous avons des contacts; parce que l'on peut-être invités, parce qu'on nous donne un doctorat honorifique, on peut venir jusqu'à vous. Mais je pense que cette Église a d'autres représentants de ce qui se vit vraiment là-bas. Je vous rapporte une expérience plus ou moins personnelle. II y a trois ou quatre ans, j'étais allé rencontrer une équipe de pastorale formée autour de prêtres. II y avait parmi eux un canadien et des religieuses. A cet endroit, les personnes connaissent toutes sortes de violence: terrorisme qu'on ne peut pas accepter, violence de la drogue et même violence de l'armée. Nous avons passé une nuit à discuter. L'équipe pastorale me présentait ses problèmes, et j'ai tâché de leur suggérer de faire ceci: "Non, non, nous avons déjà essayé, ça ne marche pas". "Mais faites ceci». "Ca ne marche pas non plus". Alors vers quatre heures du matin, je leur ai dit: “Écoutez, je suis vraiment ému. Je me permets de vous raconter une histoire. Au moment où l'avion quitte l'aéroport, le pilote communique avec la tour de contrôle pour lui signifier que l'appareil connait des difficultés. «ll y a ce problème-ci». Le préposé à la tour de contrôle lui dit: "fais ceci». - "Non je peux pas pour telle raison". “Fais cela, va vers la mer». - "Je ne peux pas, je n'ai pas d'essence, 
etc.". Alors le préposé de la tour de contrôle lui dit à la fin: "Dis avec moi: 'Notre-Père, qui es aux cieux...'". Cette nuit-là, j'étais à la tour de contrôle. Je leur ai suggéré de dire un "Notre-Père". Ces personnes sont inconnues; elles ne seront jamais citées dans les journaux; personne ne leur demande quels sont leurs problèmes dans l'Église. Vous savez, à côté de la souffrance de mon peuple, j'ai honte de parler de mes difficultés; c'est pour cela que je tâche de toujours échapper à cette question. Mes voisins souffrent dans mon quartier parce qu'ils ne peuvent pas apporter de la nourriture à leurs enfants. Cette équipe pastorale souffre. Son travail pastoral, à vue humaine, n'est pas efficace, parce que les situations sont tellement difficiles.

Quand je rencontre de ces personnes, je nourris ma foi et mon espérance. Une Église qui est capable de faire naître des équipes comme celle-là a une très grande générosité. Je ne prétends pas que l'ensemble de l'Église latino-américaine soit engagé dans la voie de Medellin, qu'elle soit tout entière solidaire des pauvres, Je veux simplement dire qu'il y a quand même de trés belles choses qui se font, non pas nécessairement à cause de la théologie de la libération, bien qu'il faille reconnaître que ce soit un facteur important, mais à cause de la générosité. Des gens qui viennent dans mon pays pour me voir - je fais partie aujourd'hui du tourisme ecclésial dans mon pays - me demandent quel impact a eu la théologie de la libération en Amérique latine. Je réponds toujours la même chose: "Vous savez, la réponse ne m'intéresse pas; ce qui m'intéresse, c'est l'impact de l'Évangile et de la présence des chrétiens dans le processus de libération du peuple latino-américain. Et à l'intérieur de cela, il y a une petite part pour la théologie de la libération". En disant cela, je ne prétends pas prendre une élégante distance par rapport à la théologie de la libération. Je continue d'y travailler; je pense que c'est un instrument utile, mais à sa place, pour favoriser des engagements concrets dans une Église qui doit être de plus en plus engagée avec les pauvres. Je pense que cette théologie vient finalement du contraste entre une réalité marquée par la pauvreté et donc par la mort, et la nécessité d'annoncer la résurrection de JésusChrist. Jamais, dans la Bible, la résurrection de Jésus est appelée un miracle, car le mot miracle est trop petit pour parler de la résurrection de Jésus. Elle est plutôt la victoire sur la mort.

Vous vous rappelez ce texte de l'Ancien Testament que saint Paul répète: «Mort où est ta victoire?». Toute fête chrétienne est une moquerie de la mort pour affirmer que la mort n'est pas le dernier mot de l'histoire. La vie est le dernier mot de l'existence humaine. Je pense 
que c'est cela qui est en procès dans cette Église latino-américaine. Pour cette raison, la réflexion théologique a une valeur parce qu'elle est soutenue par la vie d'une Église ou d'une grande partie de cette Église. Autrement, elle n'aurait pas beaucoup de sens en dehors du monde intellectuel.

Je termine d'une façon très peu académique et je vous demande simplement de prier pour mon continent. Mais si vous tenez compte des pauvres du monde, vous êtes déjà en condition de trouver votre place dans cette option préférentielle pour les pauvres. 